\title{
Optimization Analysis of Sustainable Solar Power System for Mobile Communication Systems
}

\section{Mohammed H. Alsharif ${ }^{1}$, Raju Kannadasan², Amir Y. Hassan ${ }^{3}$, Wael Z. Tawfik ${ }^{4}$, Mun-Kyeom Kim ${ }^{5, *}$, Muhammad Asghar Khan ${ }^{6}$ and Ahmad A. A. Solyman}

\author{
${ }^{1}$ Department of Electrical Engineering, College of Electronics and Information Engineering, Sejong University, \\ 209 Neungdong-ro, Gwangjin-gu, Seoul, 05006, Korea \\ ${ }^{2}$ Department of Electrical and Electronics Engineering, Sri Venkateswara College of Engineering, \\ Sriprumbudur, Chennai, 602117, India \\ ${ }^{3}$ Department of Power Electronics and Energy Conversion, Electronics Research Institute, Giza, 12622, Egypt \\ ${ }^{4}$ Department of Physics, Faculty of Science, Beni-Suef University, Beni-Suef, 62511, Egypt \\ ${ }^{5}$ Department of Energy System Engineering, Chung-Ang University, 84 Heukseok-ro, Dongjak-gu, Seoul, 156-756, Korea \\ ${ }^{6}$ Department of Electrical Engineering, Hamdard University Islamabad, 44000, Pakistan \\ ${ }^{7}$ Department of Electrical and Electronics Engineering, Istanbul Gelisim University, Avc1lar, 34310, Turkey \\ *Corresponding Author: Mun-Kyeom Kim. Email: mkim@cau.ac.kr \\ Received: 04 August 2021; Accepted: 07 September 2021
}

\begin{abstract}
Cellular mobile technology has witnessed tremendous growth in recent times. One of the challenges facing the operators to extend the coverage of the networks to meet the rising demand for cellular mobile services is the power sources used to supply cellular towers with energy, especially in remote. Thus, switch from the conventional sources of energy to a greener and sustainable power model became a target of the academic and industrial sectors in many fields; one of these important fields is the telecommunication sector. Accordingly, this study aims to find the optimum sizing and technoeconomic investigation of a solar photovoltaic scheme to deploy cellular mobile technology infrastructure cleanly and sustainably. The optimal solarpowered system is designed by employing the energy-balance procedures of the HOMER software tool. The problem objective is considered in terms of cost, but the energy system is constrained to meet the power demand reliably. Process simulations were performed to determine the optimum sizing, performance and monetary cost of the power system, using long-term meteorological datasets for a case study site with defined longitude $\left(31^{\circ} 25^{\prime} \mathrm{E}\right)$ and latitude $\left(30^{\circ} 06^{\prime} \mathrm{N}\right)$. From the observed results, the total net present cost (NPC) of the proposed system is $\$ 28,187$. Indeed, these outcomes can provide profound economic, technical, and ecological benefits to cellular operators. It also ensures a sizeable reduction in greenhouse gas that supports sustainable green wireless network (WN) deployment in remote areas.
\end{abstract}

Keywords: Wireless networks; green wireless networks; green communications; sustainability; OPEX 


\section{Introduction}

Cellular mobile technology has witnessed tremendous growth in recent times. The rising acceptance and accessibility of mobile broadband services are motivating a change in the engagement patterns of mobile users from basic voice to data-centric services. One of the challenges facing the operators to extend the coverage of the networks to meet the rising demand for cellular mobile services is the power sources used to supply cellular towers with energy, especially in remote areas. Accordingly, the growing demand for a sustainable energy system has made alternative power sources a promising field of investigation due to sustainability with negligible carbon emissions [1]. The renewable energy systems architecture can supply the required energy for the deployment of cellular mobile technology infrastructure.

Mobile base stations (BSs) are the key consumers of the energy used by the operators, e.g., around $57 \%$, as mentioned in [2]. WNOs (wireless network operators) have recently concentrated on mass data transmission levels with great radio coverage to meet the subscriber's demands. Consequently, WNO outstretched their BS numbers pointedly to distribute more excellent data rates to numerous wireless expedients that ensure higher accessibility in point nodes [3]. Therefore, a steep rise is perceived in energy consumption and operational expenditure (OPEX) [4]. In recent times, the immense integration of IoT devices is performed with wireless access grids that led to vast energy consumption.

Further, it is expected to rise immensely in the impending days due to the different sorts of IoT devices [5]. Therefore, the chief concentrations of WNOs involved providing great data rates while considering energy-effectual concerns [6]. Furthermore, the snowballing qualms of the WNOs about higher energy consumption rates forced them to increase the OPEX savings and increase the attentiveness of global warming concerns and other environmental glitches [7]. Hence, the inventors and researchers are concentrated on finding the optimal methodologies to significantly reduce the energy consumption amount for the WNOs while preserving the anticipated data amenities [8].

The researchers concentrate on several distinctive methodologies to decrease the energy consumption rate in WNs, notably effectual usage of radio transmission practice, energy-efficacy hardware modules, discerning operation of such modules, positioning heterogeneous cells, and employing renewable energy sources (RESs) [9]. Over the long term, RESs play the most attractive role in various domains due to their cost-effective applications of scheming energy-efficient WNs in the existing structure [10]. Specifically, the most lucrative regions for PV-based BSs are considered from a low latitudes zones [2]. More often, the anticipated zone for PV-enabled BSs is in the mid-latitude between 30 o north and south. Therefore, this work scrutinizes the practicability of using PV solutions as the chief power source to meet the energy demands of cellular BSs in Egypt's off-grid locations to determine the net OPEX savings. Egypt is positioned at defined latitude $\left(30^{\circ} 06^{\prime} \mathrm{N}\right)$ and longitude $\left(31^{\circ}\right.$ $\left.25^{\prime} \mathrm{E}\right)$, measured as a superior site for solar PV generation. It has an average daily solar irradiation stretching from $2.92 \mathrm{kWh} / \mathrm{m}^{2}$ (December) to $7.44 \mathrm{kWh} / \mathrm{m}^{2}$ (June) [11].

However, the solar energy viability system depends on various factors comprising the mix of energy resources, distributed capacity, and control strategy. The HOMER model is used by developing an energy balance scheming for each hour of $8,760 \mathrm{~h} /$ year to overcome these issues. Moreover, it compares the actual load demand for every hour with generated energy. It also manages the charging and discharging features of the batteries and computes the installation and operating cost for the project's complete lifespan. Considering all these advantages, the HOMER platform is adopted to achieve the techno-economic viability of the PV-driven LTE-BS. The contributions of this work are summarized as follows: 
- Development of solar energy system for sustainable operation of modern cell sites.

- To examine, analyze, and evaluate the energy balance for the proposed powered system.

- To examine, analyze, and evaluate the feasibility of a standalone solar system to attain maximum energy harvest and cost savings to warrant both cost-effectiveness and sustainability.

The remaining of this article is organized as follows; Section 2 describes the associated literature studies. Section 3 demonstrates the system construction and mathematical modeling. Section 4 displays the study site, data, and configurations. Then, results and discussion are designated in Section 5. Section 6 concludes the work considering the observed outcomes.

\section{Related Studies}

The study [12] aimed to provide insight into the concept of mobile networks, enabling technologies, unresolved practical issues, and several deployment challenges. Network service providers have often ignored the energy efficiency for spectral efficiency, which is presumed to be a key performance metric in designing a cellular network. Even more, the importance of energy efficiency has been realized due to the increased ecological and economic effects of cellular networks [13]. Thus, the energy-saving problem has been identified as a significant criterion in the design of future WNs.

Several studies have considered the capacity and efficiency problems of future wireless networks for sustainable energy management. The issues related to environmental concerns, high-power consumption, and insufficient energy-saving techniques are escalating rapidly in communication technologies. An insight into existing energy sustainable paradigms geared at obtaining highly sustainable and cost-effective networks was presented in [14]. The authors proposed using energy harvesting hardware as an innovative candidate to minimize the OPEX and environmental impact. Strategies to optimize the overall power consumed in a large-scale HCN without degrading network performance were investigated in [15]. Although the energy efficiency is greatly improved, the benefits come at the cost of a non-negligible network throughput degradation, which is suboptimal, mainly in real-time applications.

Authors in [16] focused on optimizing the energy efficiency in the backhaul of LTE networks. A backhaul traffic algorithm was explored, and the performance of the light, heavy, and green coordinated multi-points with and without the backhaul algorithm was investigated. A simulation result showed that the proposed algorithm green coordinated multi-point with backhaul traffic is the most efficient algorithm. Renewable-energy-powered cellular BSs to make cellular networks sustainable and greener by minimizing the total consumed energy and OPEX in mobile communication systems were investigated in [17]. Energy-efficient solutions drawn from intuitive study and observations were presented to address the challenges militating against the widespread deployment of the proposed green communication initiative-renewable-energy powered BSs. The author in [16] considered the viability of using a PV system to satisfy the power demand of an $\mathrm{HCN}$ in place of public electric grids using the HOMER model. The simulation results demonstrated that the PV system is well-adapted to supply the load requirements of the BSs in HCNs and can satisfactorily address the sustainability issues of power resources by providing greener and cost-effective energy. The authors in [18] posited that OPEX and carbon emissions are the main bottlenecks for cellular network operators. To alleviate this challenge and guarantee cost-effectiveness, sustainability, and reliability, the authors investigated the viability of a PV system to supply the required energy to remote LTE-MBSs in off-grid sites with excellent solar energy potential. The HOMER software was employed to decide the techno-economic feasibility and the optimum criteria of the PV system. The simulation outcomes indicated that the PV-powered arrangement can substantially supply the energy requirements of the cellular BSs while 
enabling a reduction in both the OPEX and greenhouse gases. However, further research is required on low-cost optimization models.

Nevertheless, more comprehensive power models and advanced optimization techniques are currently needed. In this study, the optimum size and techno-economic examination of a PV system. The objective is to switch from the typical grid diesel hybrid to a greener and sustainable power alternative. The solar system is expected to satisfy the energy requirement of the cellular infrastructure at reduced cost with a high renewable portion.

\section{System Design and Mathematical Modeling}

The architecture and model of the hybrid energy system, as presented in Fig. 1. The series and parallel connection PV panels are constructed considering the required voltage and current ratings for the PV panel. A battery bank or battery management system stores the excess energy from PV as a backup which can be utilized during non-sunny periods, notably at night (load shedding hours), ensuring reliability and the maximum energy that allows the share of the total energy of the battery bank. The DC bus, which maintains the constant voltage, supplies the power to the cellular BS. Furthermore, a DC/AC converter is used to convert the energy to AC which supplies the power to the AC load (Air conditioner).

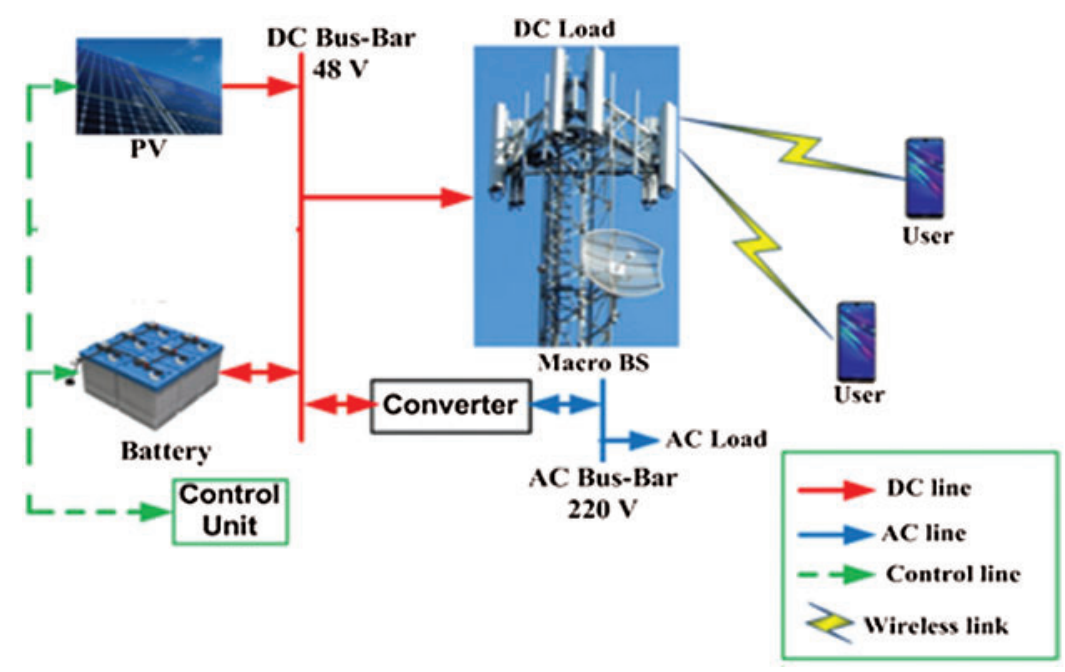

Figure 1: Architecture of proposed mixed power system for cellular sites

This backhaul network configuration has different sub-units such as DC-DC Power supply, radiofrequency (RF), multiple transceivers (TRXs), power amplifier (PA), Base band (BB) and cooling systems. Specifically, TRXs encompass PA that amplifies the signal power received from the Base band units. Further, the internal processing and programming are performed using base band units. The BS (cellular) comprises several equipment and components and can be adapted to communicate with cellular modules. Also, a comprehensive description of the BS modules can be referred in [2]. Furthermore, the energy consumption levels of various components of the BS (macro) module with a $2 \times 2$ multi-input and multi-output (MIMO) antenna planning with three segments are presented in Tab. 1. 
Table 1: Power consumption ranges of various hardware parts at a bandwidth equal to $10 \mathrm{MHz}$ [19]

\begin{tabular}{lcll}
\hline Elements & Parameters & Unit & $\begin{array}{l}\text { Power } \\
\text { consumption }\end{array}$ \\
\hline PA & $P_{P A}^{D C}$ & Watts & 102.60 \\
RF & $P_{R F}^{D C}$ & Watts & 10.90 \\
BB & $P_{B B}^{D C}$ & Watts & 14.80 \\
Loss factor 1 $\left(\sigma_{D C}\right)$ & & $\%$ & 06.00 \\
Loss factor 2 $\left(\sigma_{c o o l}\right)$ & $P_{P A}^{D C}+P_{R F}^{D C}+P_{B B}^{D C}$ & Watts & 10.00 \\
Total power/TRX $=\frac{151.65}{\left(1-\sigma_{D C}\right)\left(1-\sigma_{c o o l}\right)}$ & & 06.00 \\
No. of transceivers $N_{T R X}=N_{S e c t} \times N_{A n t} \times N_{C a r r}$ & & 909.93 \\
Total power of the BS $P_{B S}=N_{T R X} \times$ Total & Watts \\
power/TRX & & \\
\hline
\end{tabular}

\subsection{Photovoltaics Panels}

It consists of numerous interconnected solar cells (both series and parallel connections) to design PV modules. The developed module produces DC power by means of shortwave irradiance on the panel surfaces. The net annual energy extraction from the solar PV arrangement $\left(E_{P V}\right)$ can be derived as follows [17]:

$E_{P V}=\mathrm{PC}_{P V} \times P S H \times D F_{P V} \times 365$ days/year,

where $P C_{P V}$ represents a peak capacity in $\mathrm{kW}$; $P S H$ signifies peak solar hour (average daily solar radiation). $D F_{P V}$ denotes a system derating factor involving the influence of temperature disparities, dust, and technical and non-technical losses that impact the output power of the PV panel.

\subsection{Battery Banks}

The capacity of the battery banks used in BS purely hinges on the depth of discharge (DOD) estimated using Eq. (2) before installation [20].

$D O D=1-\frac{S O C_{\min }}{100}$

where $S O C_{\text {min }}$ denotes the minimum magnitude of state of charge (SOC). In this work, the Trojan L16P battery modules are used because the DOD of this battery is $70 \%$, i.e., it can effectually deliver $70 \%$ of energy with a reserved capacity of $30 \%$. It is essential to compute the days of autonomy $\left(A_{\text {batt }}\right)$ to study the capability of the $100 \%$ charged batteries (energy supplying days from fully charged batteries excluding auxiliary power sources). It can be written as [20],

$A_{\text {batt }}=\frac{N_{\text {batt }} \times V_{\text {nom }} \times Q_{\text {nom }}\left(1-\frac{S O C_{\min }}{100}\right)(24 \mathrm{~h} / \mathrm{d})}{L_{\text {prim-avg }}(1000 \mathrm{Wh} / \mathrm{kWh})}$

where $N_{\text {batt }}$ and $V_{\text {nom }}$ are the number of battery modules and the nominal voltage of a single battery module (V) in the BESS, respectively; $Q_{\text {nom }}$ is the nominal capacity of a one battery unit (Ah); $L_{\text {prim, ave }}$ is the average daily BESS load in $\mathrm{kWh}$, respectively. 
It is a known fact that the lifetime of the battery modules plays a vital role. It can be anticipated considering the operating conditions of the complete system. Pointedly, the DOD of the battery module during each diurnal charge-discharge phase exhibits a leading role in the battery life, and it can be calculated as [16],

$R_{\text {batt }}=\min \left(\frac{N_{\text {batt }} \times Q_{\text {lifetime }}}{Q_{\text {thrpt }}}, R_{\text {batt } f}\right)$

where $Q_{\text {lifetime }}$ signifies the lifetime of an individual battery unit in kWh; $Q_{\text {thrpt }}$ symbolizes the annual battery throughput (kWh/year); $R_{\text {batt }}$ denotes float lifetime of batteries in years.

\subsection{Inverter}

The net inverter capacity $\left(C_{i n v}\right)$ can be estimated using the below equation [21],

$C_{i n v}=\left(\frac{L_{A C}}{\eta_{i n v}}\right) \times \sigma_{s f}$

where $L_{A C}$ signifies the available AC load (maximum); $\eta_{i n v}$ denotes the inverter efficiency; $\sigma_{s f}$ represents the safety factor.

The arrangement of PV-powered base station is considered based on the following factors: the vital components that must be used in the system design; a number of components adopted; the size of the individual component. To attain an optimum PV system with lesser net present cost (NPC), the HOMER Micro-power optimization tool is adapted. The NPC covers all incurred expenditures and profits throughout the venture lifetime, and it can be computed using the below equation:

$N P C=\frac{T A C}{C R F}$

The term TAC signifies a total annualized cost representing the cost of complete arrangement in $\$ /$ year. It encompasses various costs such as operation and maintenance $(\mathrm{O} \& \mathrm{M})$ costs, initial capital (IC) costs, and replacement costs.

Similarly, $C R F$ symbolizes a recovery factor that changes $C_{N P C}$ into equal annual flow costs over a specific duration. The scale of CRF can be computed considering the total number of years $(\mathrm{N})$ and yearly interest rate $(i)$, using the below equation [16]:

$C R F(i, N)=\frac{i(1+i)^{N}}{(1+i)^{N}-1}$

The full NPC is reduced markedly owing to salvage value (S) precisely at the end of the venture lifetime, and it can be computed using the below equation;

$S=C_{\text {rep }} \frac{R_{\text {rem }}}{R_{\text {comp }}}$

where $R_{\text {comp }}$ states the lifetime of the various components (years); $R_{\text {rem }}$ signifies the remaining lifetime of the modules (years); $C_{\text {rep }}$ represents the replacement cost of the element (\$). 
This work examines to reduce the total NPC considering several constraints. Further, the objective function of the NPC can be computed using the below equation to accomplish system optimization.

$$
\min _{E_{P V}, E_{\text {Battery }, E_{\text {Losses }}, E_{B S}}} \frac{C_{T A C}}{C R F(i, N)} .
$$

The above-mentioned objective function is exposed to the succeeding constraints:

$E_{\text {Battery }}+E_{P V}>0$,

$E_{\text {Battery }}+E_{P V} \geq E_{B S}+E_{\text {Losses }}$.

$E_{\text {Battery_min }} \leq E_{\text {Battery }} \leq E_{\text {Battery_max }}$

It is essential to maintain the power generation of the available sources $\left(E_{P V}\right.$ and $\left.E_{B a t t e r y}\right)$ that should meet the BS demand $\left(E_{B S}\right)$ and losses $\left(E_{\text {Losses }}\right)$ to warrant an energy balance between demand and generation.

\section{Study Site, Data, and Configurations}

The anticipated region for PV-powered BSs is usually in the mid-latitudes between $30^{\circ}$ north and south. Specifically, low latitudes are recognized as the most lucrative regions for solar PV-based BSs [2]. Considering all the inferences mentioned above, researchers have insufficiently described the total OPEX savings due to RESs. Therefore, we are using solar power solution for LTE-BSs in Oman's offgrid locations to determine the net OPEX savings in this study. Egypt is positioned at latitude (30 $06^{\prime}$ $\mathrm{N})$ and longitude (31 $\left.25^{\prime} \mathrm{E}\right)$ [11]. Fig. 2 shows the solar radiation map of Egypt. In addition, the solar irradiance of Egypt that is considered in this study is given in Fig. 3. Moreover, the simulation study involves three major parts such as inputs, optimization, and outputs. The simulation configuration details are provided in Tab. 2.

\section{Results and Discussion}

Considering the economic factors, the net NPC is taken as $\$ 28,187$ that encompasses $4.5 \mathrm{~kW}$ solar PV modules and 64 batteries, and they are connected in 8 parallel arrangements along with an inverter of $0.1 \mathrm{~kW}$. The total energy output is observed from the designated system as $7,372 \mathrm{kWh}(4.5$ $\mathrm{kW} \times 5.28 \mathrm{~h} \times 0.85 \times 365$ days/year), which is estimated using Eq. (1). Specifically, $29 \%$ of generating energy output is increased annually, i.e., 9,509 $\mathrm{kWh}$ more, by adopting a dual-axis tracking system. Pointedly, this energy yield enhancement meets the total losses experienced in the batteries and inverter about 770 and $42 \mathrm{kWh}$, respectively. It supplies the energy to the load of about 7,972 kWh, i.e., to the macro LTE-BS, resulting in excess energy of about $725 \mathrm{kWh}$ annually, i.e., $7.62 \%$ of the total energy production. Fig. 4 demonstrates the monthly average energy generation by the PV system. The maximum energy and minimum energy generations were observed to occur in June and December, respectively. 


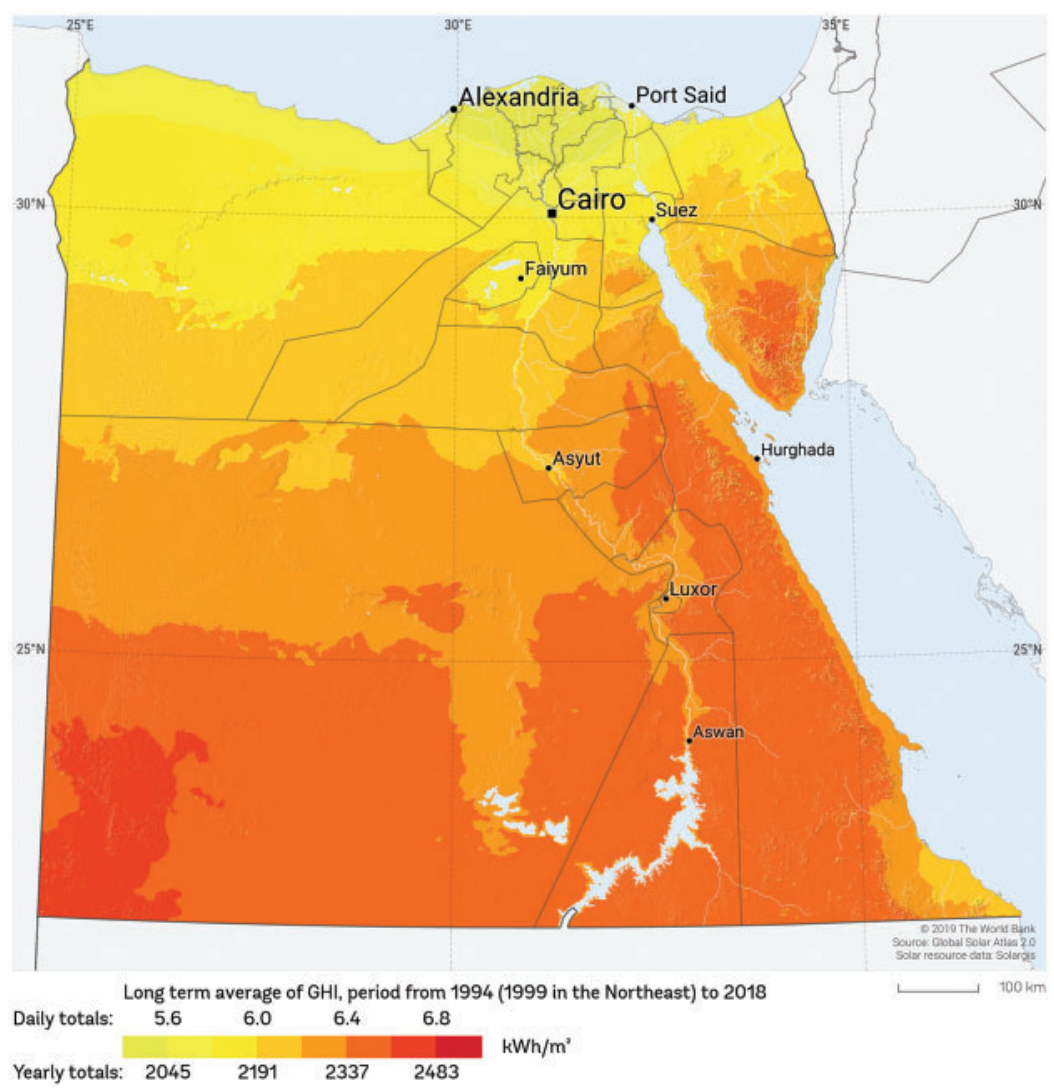

Figure 2: Solar radiation map of Egypt

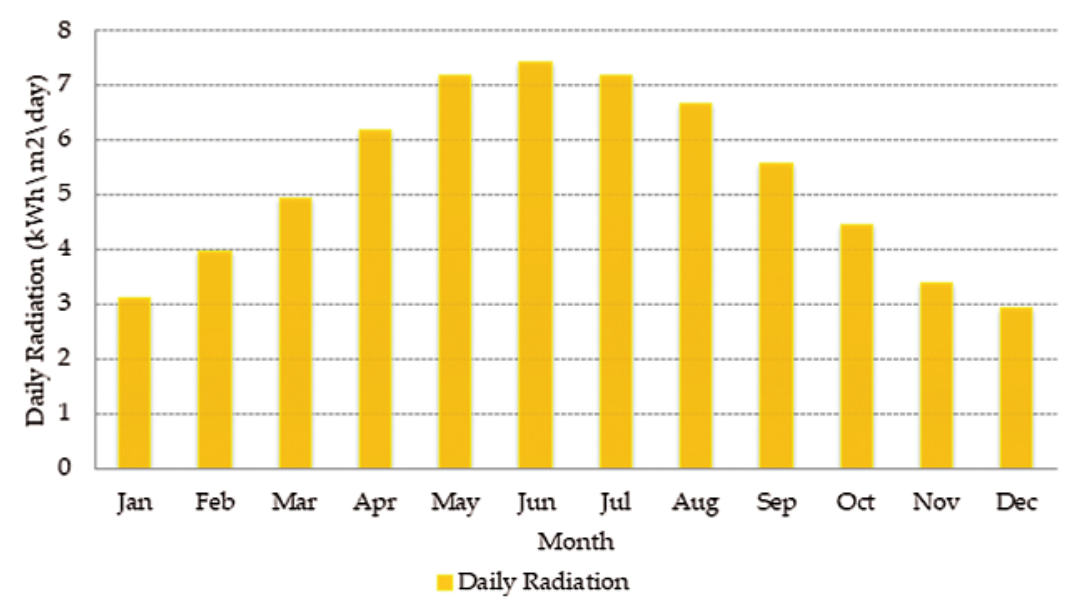

Figure 3: Solar irradiance of Egypt (monthly average)

The optimum capacity of the battery bank that is computed by the HOMER software for the designated system is 64 units. Pointedly, the voltage rating of a single battery is estimated to be $6 \mathrm{~V}_{\mathrm{dc}}$ (Trojan L16P), and its capacity is determined as $360 \mathrm{Ah}$. To attain the required ratings, a total of 64 batteries are associated in both series and parallel that could maintain the constant voltage of $48 \mathrm{~V}_{\mathrm{dc}}$ 
connected in the bus bar. The roundtrip efficiency of the battery reached $85 \%$, calculated between the ratios of yearly energy output and input, i.e., 4,369 and 5,139 kWh, respectively. Approximately, the battery bank feeds the energy to the load for nearly $106 \mathrm{~h}$, notably during the failure of the PV array. Based on the inferences from Fig. 4, it is perceived that the PV module generates the energy minimally in December. Therefore, it is forcibly allowed the battery banks to supply maximum power in December to compensate for the diminishing energy support of the PV modules, as illustrated in Fig. 5. Accordingly, the stored energy of the battery bank has reduced into the lowest range, and the state of charge (SOC) stretched to $33 \%$, as presented in Fig. 6. Also, the average hourly energy production of the solar PV, energy storage in battery, and surplus electricity for 12 months are displayed in Fig. 7.

Table 2: Configuration of the simulation study

\begin{tabular}{lll}
\hline Elements & Parameters/factors & Values \\
\hline Control factors & Interest rate-annual (Jul. & $8.5 \%$ \\
& Proj) & \\
Inverter & Sizes considered & Ten years \\
& & $0.1,0.2,0.3$, \\
& Efficiency & $0.4 \mathrm{~kW}$ \\
& Operational lifespan & $95 \%$ \\
& IC price & 15 years \\
& Replacement cost & $\$ 0.4 /$ Watt \\
& O \& M price per year & $\$ 0.4 /$ Watt \\
Solar-PV & Sizes considered & $2,0.01 /$ Watt \\
& & $4.5,5 \mathrm{~kW}$ \\
& Operational lifespan & 25 years \\
& Efficiency & $85 \%$ \\
& IC price & $\$ 1 /$ Watt \\
& Replacement cost & $\$ 1 /$ Watt \\
& O \& M price/year & $\$ 0.01 /$ Watt \\
& Number of batteries & $24,32,40$, \\
& & 64,72 \\
& Round trip efficacy & $85 \%$ \\
& Minimal operational lifetime & Five years \\
& IC & $\$ 300$ \\
& Replacement & $\$ 300$ \\
& O \& M price/year & $\$ 10$ \\
\hline
\end{tabular}

The total inverter capacity is considered as $0.1 \mathrm{~kW}$, and its efficacy is figured between the input $(837 \mathrm{kWh})$ and output energy $(795 \mathrm{kWh})$ annually and perceived as $95 \%$. Also, the net operating hours are found to be $8,759 \mathrm{~h} /$ year $(24 \mathrm{~h} \times 365$ days/year $)$. Fig. 8 presents a cash flow summary of the project. The details of costs based on each component in the proposed system are given as follows. 


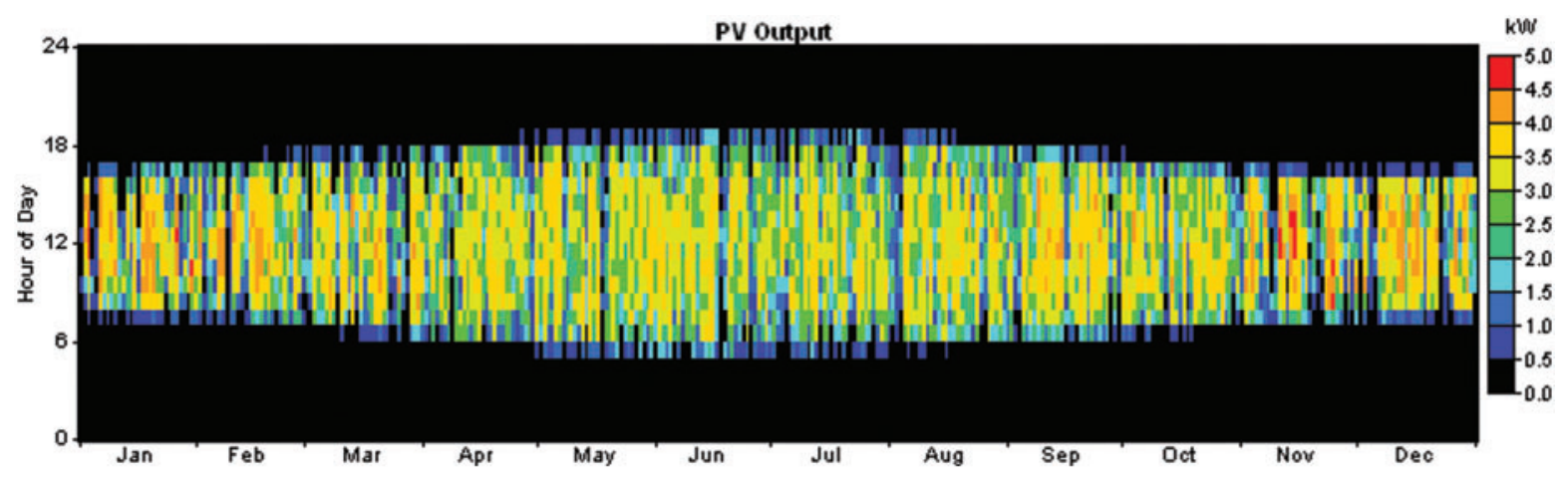

Figure 4: Average PV output power (month wise)

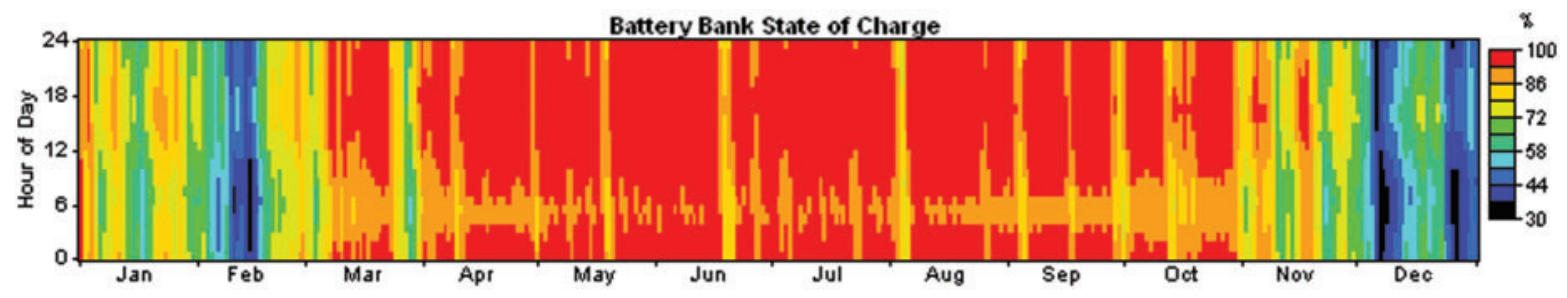

Figure 5: Maximum and minimum SOC (seasonal statistics)

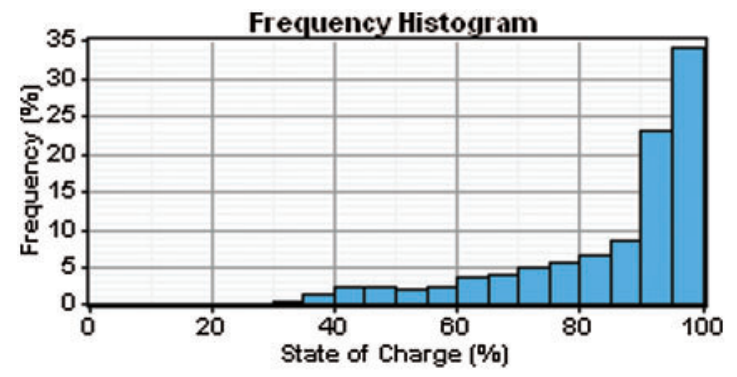

Figure 6: Battery bank state of charge (monthly statistics)

The total capital of the PV array is $\$ 4,500$, which is computed as $4.5 \mathrm{~kW}$ (size) $\times \$ 1,000 / 1$ $\mathrm{kW}$ (cost). The O\&M cost of the PV array considered low is $\$ 450$ which is computed as $4.5 \mathrm{~kW}$ $($ size $) \times \$ 10 / 1 \mathrm{~kW}($ cost $) \times 10$ years (macro LTE-BS lifetime). Due to the higher lifetime of PV panels ( 25 years) compared with project lifetime (10 years); the replacement cost of the solar system is zero. Further, the PV array's salvage value is $\$ 2,400$, calculated using Eq. (8), which is the highest rate among other modules. Thus, the normal net NPC of PV array is computed by addition of aggregated cash flows/year and found to be $\$ 2,550$, i.e., $\$ 4,500$ (capital cost) $+\$ 450$ (O\&M cost) - \$2,400 (salvage)

Further, the total capital of the battery is $\$ 19,200$, which is computed as 64 (units) $\times \$ 300 / 1$ unit (cost). The O\&M price of the battery units is considered high at about $\$ 6,400$, which is computed as 64 (units) $\times \$ 10 / 1$ unit $($ cost $) \times 10$ years (macro LTE-BS lifetime). Also, the battery pack's replacement cost is considered zero since the battery bank has a lifespan of 10 years, i.e., the macro LTE-BS lifetime. Thus, the normal net NPC of battery bank is $\$ 25,600$, i.e., $\$ 19,200$ (capital cost) $+\$ 6,400$ (O\&M cost) $-\$ 0$ (salvage). 


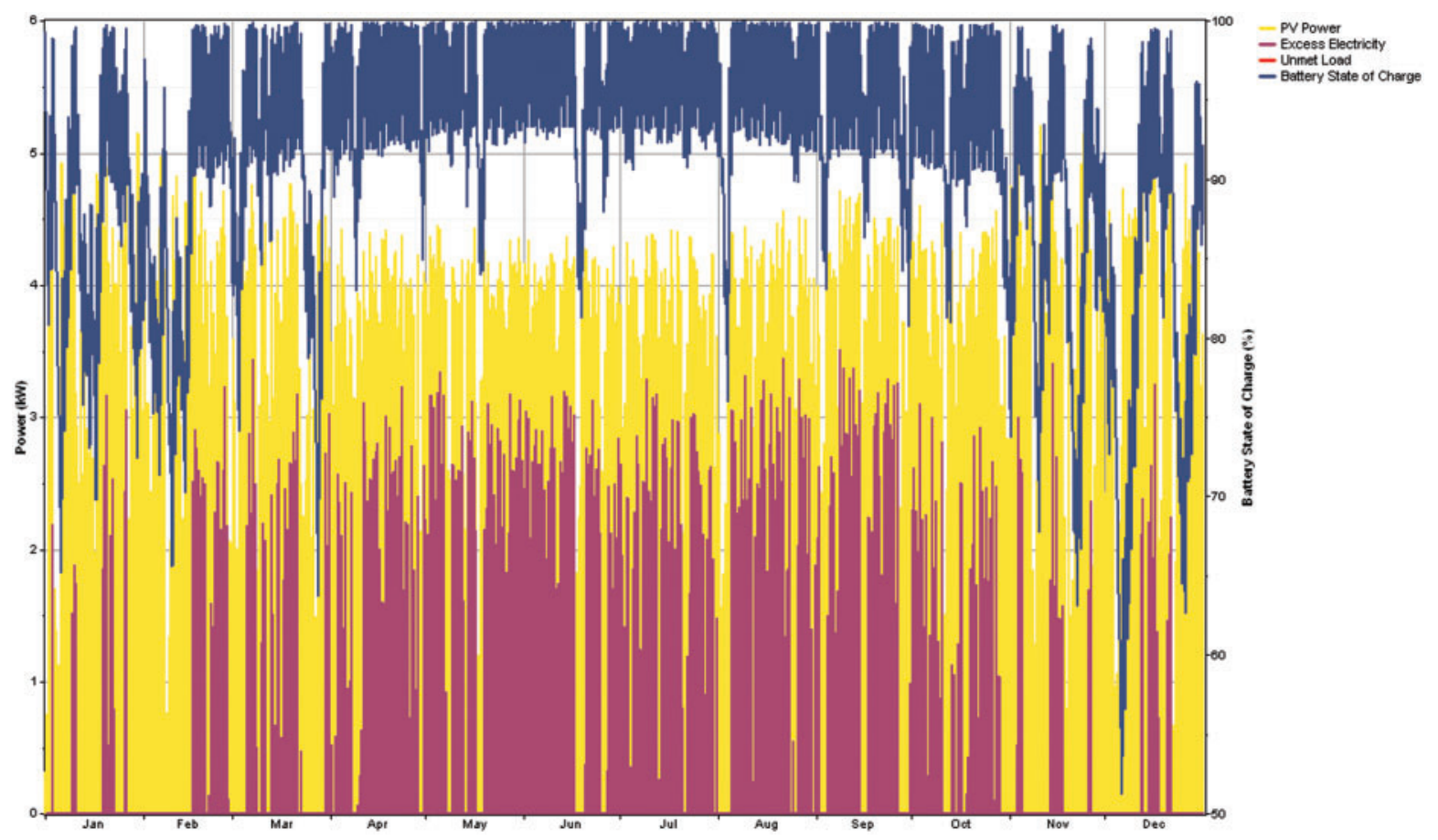

Figure 7: Average PV array, battery bank, and excess electricity output (hourly)

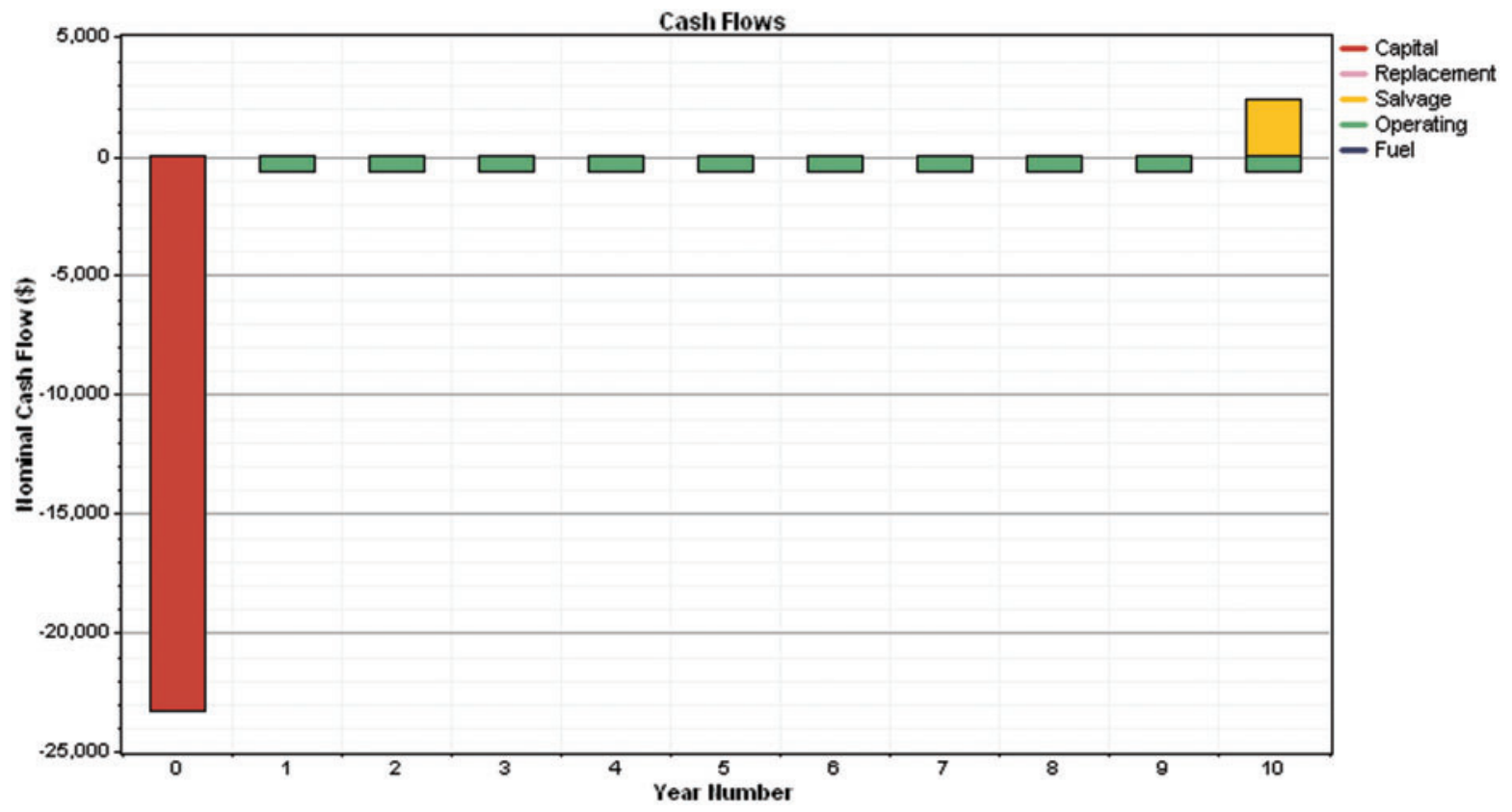

Figure 8: Summary of cash flow of the PV array (project lifespan)

Similarly, the total capital of the inverter is evaluated and found to be $\$ 40(0.1 \mathrm{~kW}$ (size) $\times \$ 400 / 1$ $\mathrm{kW}($ cost)). The O\&M price of the inverter is $\$ 10$, which is computed as $0.1 \mathrm{~kW}($ size $) \times \$ 10 / 1 \mathrm{~kW} \times 10$ years (macro LTE-BS lifespan). The replacement cost again becomes zero because the inverter has a 
lifespan of 15 years higher than the project lifespan or macro LTE-BS lifetime of 10 years. The salvage value is $\$ 13$. Thus, the normal net NPC of inverter is found to be $\$ 37$, i.e., $\$ 40$ (capital cost) $+\$ 10$ (O\&M cost) - \$13 (salvage).

Consolidating the above inferences, the total NPC for the proposed solar system is $\$ 28,187$. Notably, the battery bank evidently represents the bulk cost compared with other components. Nonetheless, this cost depends on the battery numbers used in the system. In this study, the optimum number of batteries was found to be 64 evaluated through HOMER software. Thus, the number of batteries used in the proposed case can be reduced significantly. However, the load autonomy declines, and it is considered a vital concern, specifically in off-grid areas. Further, the replacement cost of the entire cost is zero-based on the observed results because all the significant components, notably battery bank, PV panes, and inverter unit, have a higher lifetime than the project lifespan.

\section{Conclusions}

This work proposed a framework for an energy-efficient RES-based cellular network for Egypt off-grid sites using a PV module that acts as the primary and standalone source for the base stations to minimize the OPEX. Moreover, the architecture of the system, energy yield investigation, and economic exploration was discussed. From the observed results, it is revealed that the suggested PVbased system could possibly meet the net energy demand of the macro LTE-BS. Furthermore, the battery bank could meet the power demand of the macro LTE-BS load autonomy for $106 \mathrm{~h}$; this figure is considered an adequate time to fix the solar PV array during malfunction scenarios. Regarding the economic aspect, the simulation outcomes exhibited that the anticipated solar PV system could achieve OPEX savings with a reliable energy supply. These results indicate that the PV-based power system is a good and effective choice for wireless network operators.

Funding Statement: This research was supported by the Korea Electric Power Corporation (Grant number: R19XO01-37). This research was also supported by Basic Science Research Program through the National Research Foundation of Korea (NRF) funded by the Ministry of Education (2020R1A2C1004743).

Conflicts of Interest: The authors declare that they have no conflicts of interest to report regarding the present study.

\section{References}

[1] A. Srivastava, M. Gupta and G. Kaur, "Energy efficient transmission trends towards future green cognitive radio networks (5GProgress, taxonomy and open challenges," Journal of Network and Computer Applications, vol. 168, no. 2, pp. 102760-102775, 2020.

[2] M. H. Alsharif, J. Kim and J. H. Kim, "Green and sustainable cellular base stations: An overview and future research directions," Energies, vol. 10, no. 5, pp. 587-607, 2017.

[3] M. H. Alsharif, R. Nordin and M. Ismail, "Classification, recent advances and research challenges in energy efficient cellular networks," Wireless Personal Communications, vol. 77, no. 2, pp. 1249-1269, 2014.

[4] K. Yahya and O. Alomari, "A new maximum power point tracking algorithm based on power differentials method for thermoelectric generators," International Journal of Energy Research, vol. 16, no. 2, pp. 760-785, 2020.

[5] M. H. Alsharif, S. Kim and N. Kuruoğlu, "Energy harvesting techniques for wireless sensor networks/radiofrequency identification: A review,”Symmetry, vol. 11, no. 7, pp. 865-885, 2019. 
[6] M. H. Alsharif, J. Kim and J. H. Kim, "Energy optimization strategies for eco-friendly cellular base stations," Energies, vol. 11, no. 6, pp. 1500-1520, 2018.

[7] Z. Niu, "Green communication and networking: A new horizon," IEEE Transactions on Green Communications and Networking, vol. 4, no. 3, pp. 629-630, 2020.

[8] M. S. Hossain, A. Jahid, K. Z. Islam, M. H. Alsharif, K. M. Rahman et al., "Towards energy efficient load balancing for sustainable green wireless networks under optimal power supply," IEEE Access, vol. 8, no. 2, pp. 200635-200654, 2020.

[9] M. Hossain, A. Jahid, K. Islam, M. H. Alsharif and M. Rahman, "Multi-objective optimum design of hybrid renewable energy system for sustainable energy supply to a green cellular networks," Sustainability, vol. 12, no. 9, pp. 3536-3556, 2020.

[10] M. H. Alsharif, "Comparative analysis of solar-powered base stations for green mobile networks," Energies, vol. 10, no. 8, pp. 1208-1258, 2017.

[11] A. Gastli and Y. Charabi, "Solar electricity prospects in Oman using GIS-based solar radiation maps," Renewable and Sustainable Energy Reviews, vol. 14, no. 2, pp. 790-797, 2010.

[12] G. Auer, V. Giannini, C. Desset, I. Godor, P. Skillermark et al., "How much energy is needed to run a wireless network?," IEEE Wireless Communications, vol. 18, no. 5, pp. 40-49, 2011.

[13] M. H. Alsharif and J. Kim, "Optimal solar power system for remote telecommunication base stations: A case study based on the characteristics of South Korea's solar radiation exposure," Sustainability, vol. 8, no. 9, pp. 942-962, 2016.

[14] M. H. Alsharif and J. Kim, "Hybrid off-grid SPV/WTG power system for remote cellular base stations towards green and sustainable cellular networks in South Korea," Energies, vol. 10, no. 1, pp. 9-29, 2016.

[15] M. H. Alsharif, "Optimization design and economic analysis of energy management strategy based on photovoltaic/energy storage for heterogeneous cellular networks using the HOMER model," Solar Energy, vol. 147, no. 1, pp. 133-150, 2017.

[16] M. H. Alsharif, R. Nordin and M. Ismail, "Green wireless network optimisation strategies within smart grid environments for long term evolution (LTE) cellular networks in Malaysia," Renewable Energy, vol. 85, no. 3, pp. 157-170, 2016.

[17] S. T. Odonkor and T. Mahami, "Knowledge, attitudes, and perceptions of air pollution in Accra, Ghana: A critical survey," Journal of Environmental and Public Health, vol. 2020, no. 9, pp. 1-8, 2020.

[18] P. Mehndiratta, A. Jain, S. Srivastava and J. E. Gupta, "Environmental pollution and nanotechnology," Environment and Pollution, vol. 2, no. 2, pp. 49-54, 2013.

[19] Y. Huang, Q. Zhao, Q. Zhou and I. A. Jiang, "Air quality forecast monitoring and its impact on brain health based on big data and the internet of things," IEEE Access, vol. 6, no. 5, pp. 78678-78688, 2018.

[20] K. Bianchini, R. Alvo, D. C. Tozer and M. L. Mallory, "The legacy of regional industrial activity: Is loon productivity still negatively affected by acid rain," Biological Conservation, vol. 255, no. 3, pp. 108977108997, 2021.

[21] D. Rickerby, M. J. S. Morrison and T. O. Aly, "Materials, nanotechnology and the environment: A european perspective," Science and Technology of Advanced Materials, vol. 8, no. 2, pp. 19-30, 2007. 